\title{
المفاهيم الفلسفية والجمالية لفن الطاقة كمدل لاثراء المشغولة الخشبية المعاصرة
}

\section{Philosophical and aesthetic Concepts of Energy art As an Study to Enrich Contemporary Wood Works}

\author{
د.حامد عباس محمود سيد احمد \\ مدرس بقسم التربية الفنية تخصص اشغال الخشب \\ بكلية التربية النوعية جامعة المنوفية
}

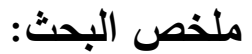

قدمت لنا الاتجاهات الفنية المعاصرة انماطا واشكالا مختلفة من الفنون من خلال تطور

الفكر الابداعي المتمثل فى المدارس والحركات والمذاهب التشكيلية الحديثة والمعاصرة ، حيث من الهن نرى نطورا واضحا فى تكوين وبناء العمل الفني داخل دراسة تشكيلية جادة ، والتي شغلت مساحة كبيرة من تفكير رواد المدارس الحديثة ، بهدف اثراء مجال الفن التشكيلي باعمال فنية

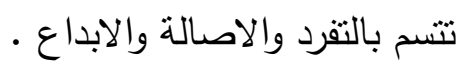

ومجال اشغال الخشب يسعي إلي مواكبة التقدم والازدهار الفنى ، بهدف الوصول الى والى

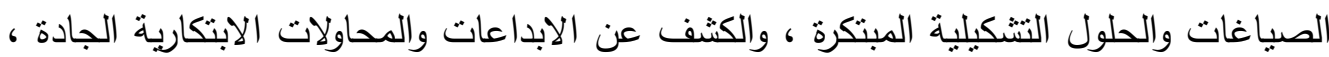

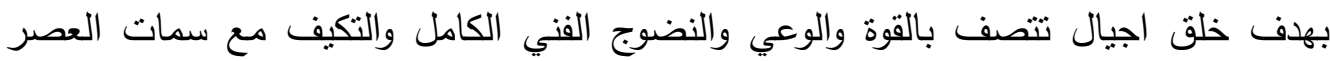
الحالي عصر السرعة والانجازات والتكنولوجيا ، فعلينا ان نبذل المزيد من الجهد والدراسة وان نستخدم احدث الاساليب التشكيلية والبعد عن كل ما هو مألوف، تقليدي والمحاولة الجادة لتقديم

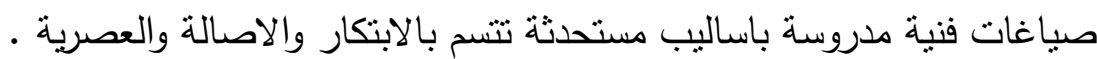
وخامة الخشب من الخامات التي تمنالك مقومات ومميزات مثل سهولة التشكيل وتقبلها لإجراء العمليات التقنية المختلفة ، والتي يمكن أن تساهم في تحقيق القيم الجمالية للششغولة

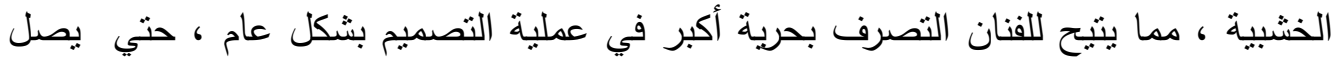
في النهاية بعمل فني ينبض بطاقته الذاتية ، والذي قد يكون له أثر كبير في توجيه نظر بـانه

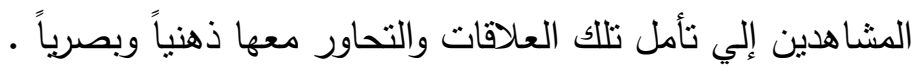


فمن خلال دراسة بعض الاعمال لفناني الطاقة ، يمكن الكثف عن القيم الجمالية للمشغولة الخشب مما يوفر للمتعلم فرصة للتجريب والممارسة ليكثف عن القيم والمفاهيم الفلسفية والجمالية لفن الطاقة ، وكيفية تتاولها برؤية مبتكرة مما يزيد فاعلية الحوار التتكيلي بين مكونات العمل الفني ، ويتيح إنتاج مشغولة خشبية تتميز بالمعاصرة . وعلي ذلك فالباحث يحاول الإفادة من جوهر المعطيات الجمالية والتشكيلية لفن الطاقة

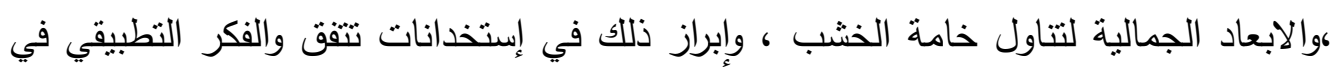
مجال اشغال الخشب ، حيث أن مجال اشغال الخشب معني بدراسة كل ما هو جديد في مجال الفن التشكيلي لطرح مداخل جديدة للتدريس ، من خلال الابعاد الفلسفية والمفاهيم التشكيلية والتقنية الحديثة ، للوصول إلي اعمال فنية معاصرة في مجال اشغال الخشب . 


\section{Abstract}

Contemporary artistic trends introduced us to different styles and forms of the arts through the development of creative thought represented in schools, movements and modern and modern plastic arts. We see a clear development in the composition and construction of the artistic work within a serious computer study, which occupied a large area of thinking of school leaders Modern art, with the aim of enriching the field of art with works of art that are unique, original and creative.

And the field of woodworking seeks to keep abreast of the progress and prosperity of art, in order to access the formulations and innovative plastic solutions, and the discovery of innovations and creative endeavors seriously, in order to create generations of strength and awareness and full technical maturity and adapt to the features of the current era era of speed and achievements and technology, We need to make more effort and study, use the latest plastic styles and keep away from everything that is familiar, traditional, and serious attempt to provide artistic formulations with innovative methods, innovative, original and modern.

And wood raw materials that have the characteristics and features such as ease of formation and acceptance of the various technical processes, which can contribute to achieve the aesthetic values of woodworking, allowing the artist to act more freely in the design process in general, Which may have a significant impact in directing viewers to the contemplation of these relations and dialogue with them mentally and visually.

Through the study of some works of energy artists, the aesthetic values of woodworking can be revealed, thus providing the learner with an opportunity for experimentation and practice to reveal the philosophical and aesthetic values and concepts of the art of energy, and how to deal with an innovative vision which increases the effectiveness of the plastic dialogue between the components of the work.

Therefore, the researcher tries to benefit from the essence of the aesthetic and formative data of the art of energy, and the aesthetic dimensions of the wood material, and to highlight it in the uses consistent with applied thought in the field of woodwork, as the field of woodwork is interested in studying everything new in the field of art to introduce new approaches to teaching, Through the philosophical dimensions and concepts of plastic and modern technology, to access the work of contemporary art in the field of woodwork 


\section{خلفية البحث: - ان}

قدمت لنا الاتجاهات الفنية المعاصرة انماطا واشكالا مختلفة من الفنون من خلال تطور

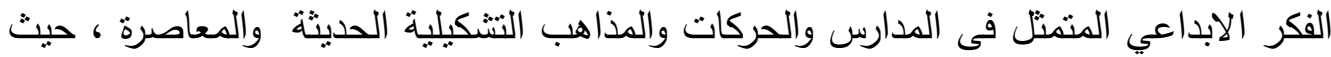
نرى تطورا واضحا فى تكوين وبناء العمل الفني داخل دراسة تشكيلية جادة ، والتي شغلت مساحة كبيرة من تفكير رواد المدارس الحديثة ، بهدف اثراء مجال الفن التشكيلي باعمال فنية

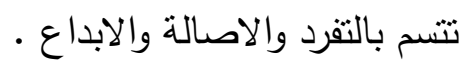

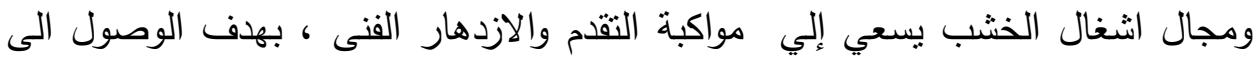
الصياغات والحلول التشكيلية المبتكرة ، والكثف عن الابداعات والمحاولات الابتكارية الجادة،

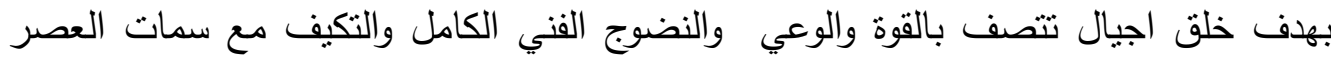
الحالي عصر السرعة والانجازات والتكنولوجيا ، فعلينا ان نبذل المزيد من الجهد و والدراسة وان فان

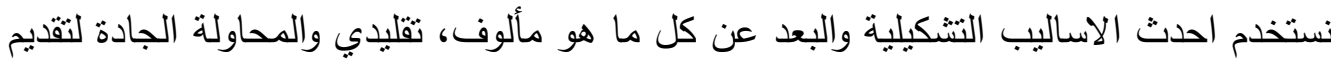

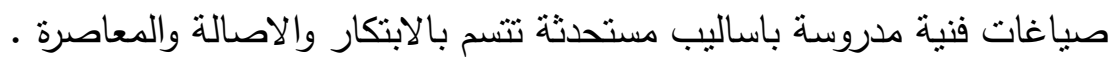

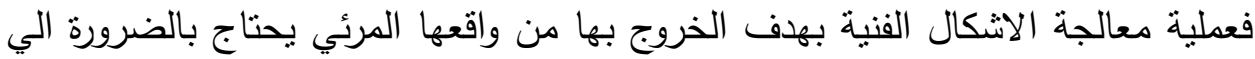
بعض الاساليب الفنية والتقنية المستحدثة لتقوم بدور الوسيط للحصول علي اشكال جديدة بكيفية

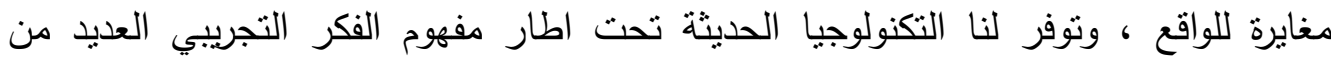

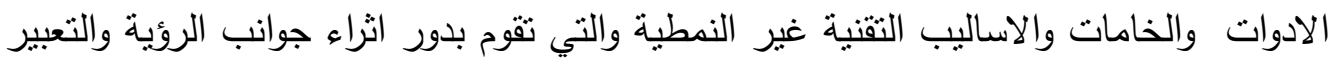

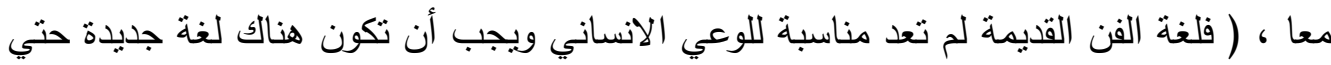

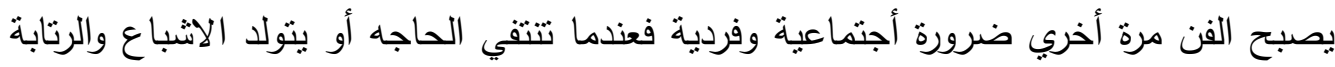

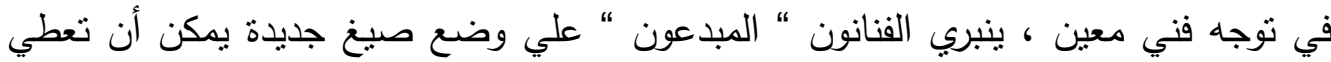

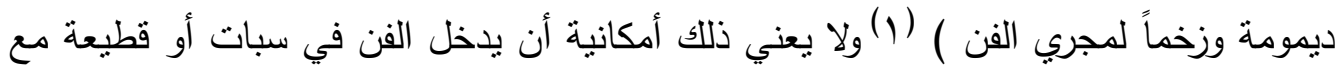

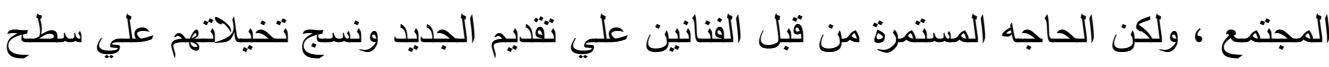
العمل الفني، وكذللك رغبة المجتمع إلي التحديث ، يمثلان النافذة التي تمنح الفن تلكئ الجمالية

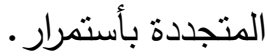

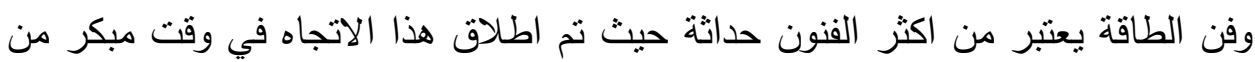

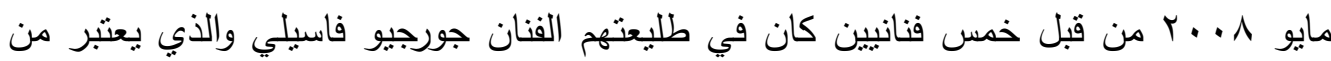

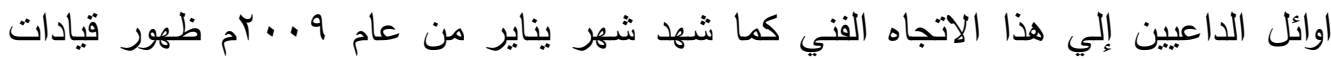

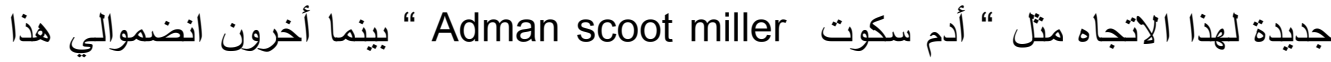

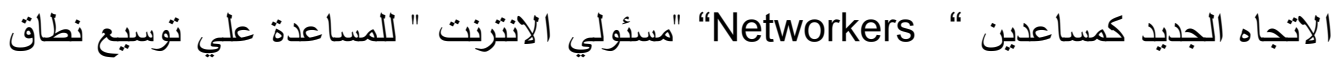

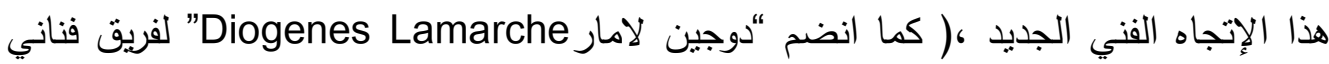

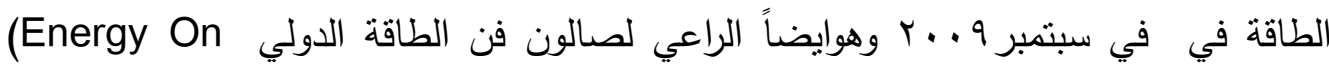
(2) Salon 2010) 
وفن الطاقة هو تصوير للطاقة باستخدام الالوان والاشكال والتكوينات لانشاء أعمال مبتكرة

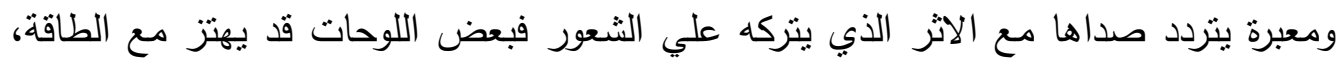

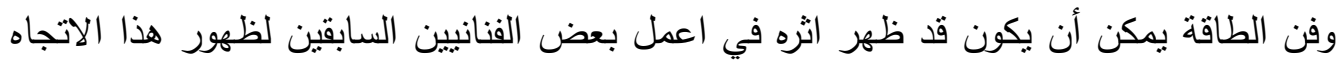

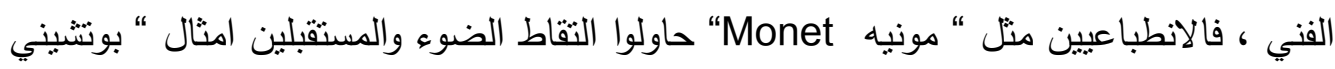
"Boccioni

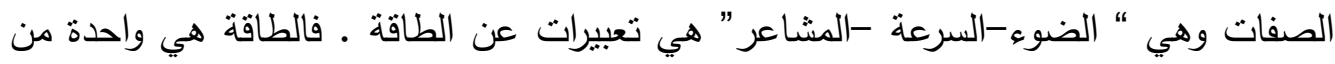

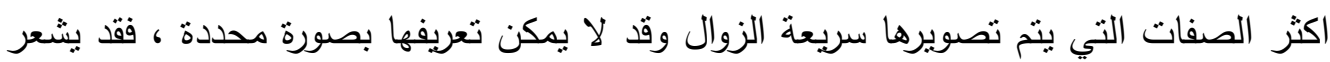

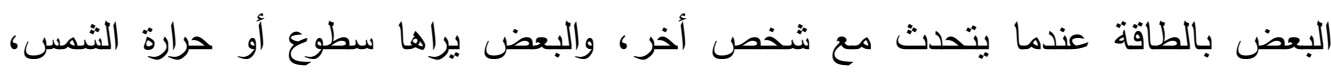
وأخرون ينظرون إليها علي أنها قوة مدمرة ، في حين يراها أخرون علي أنها نشأة الحياة فلدينا

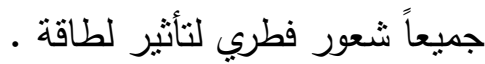
وفن الطاقة يعتمد علي ثلاثة قيم اساسية هي “لالجودة - التتوع - التطور “" علي ارضية

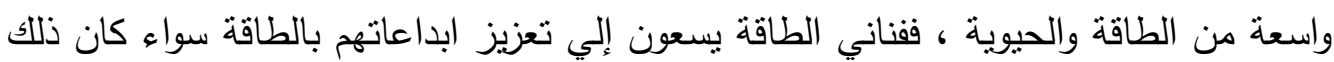

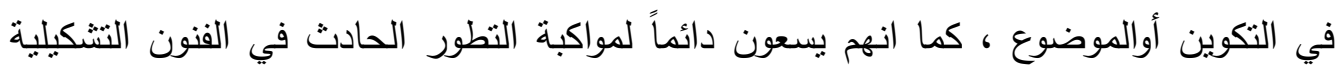

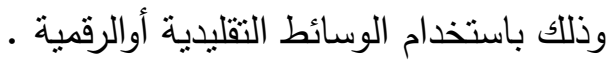
ولقد وضع فناني الطاقة خمسة معايير أساسية للاعمال الفنية التي تتتمي لهذا الاتجاه

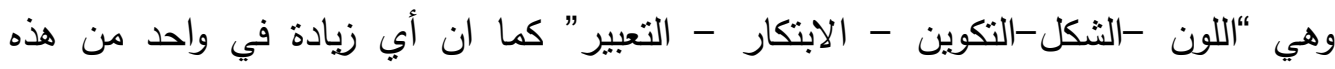

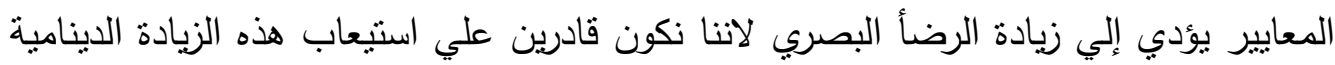

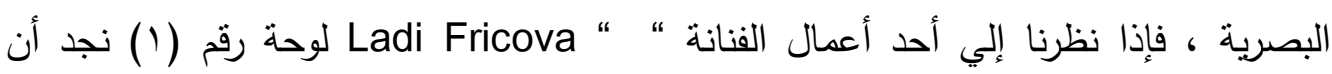

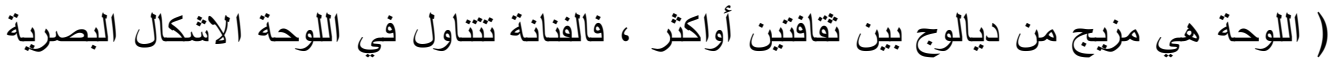

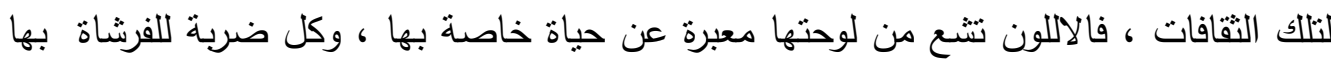

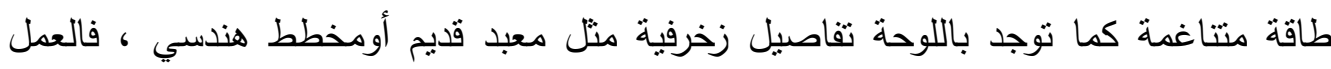

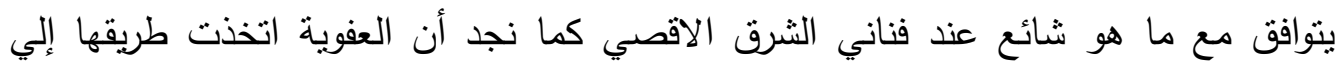

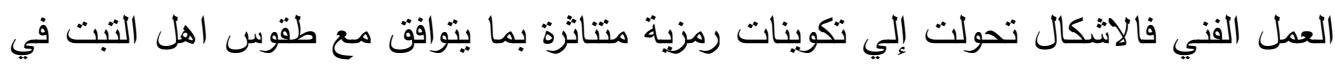

$$
\text { محاولة منها لتصوير الطاقة الداخلية للنفس البشرية )(3). }
$$

وتذكر الفنانة " Helene Kippert " (أننا جزء لا يتجزأ من بحر الطاقة غير المرئي للعين البشرية ، وفن الطاقة بالنسبة إليها يصنع مصفوفة بصرية تزيد إحساسنا للاتصال

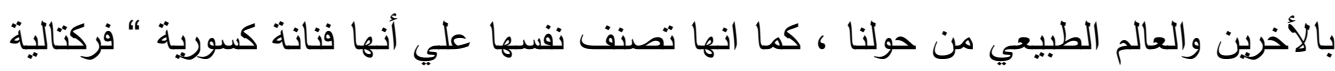

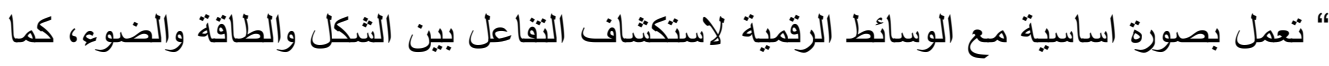

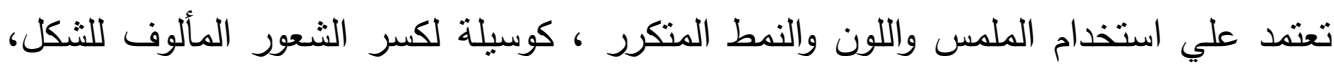

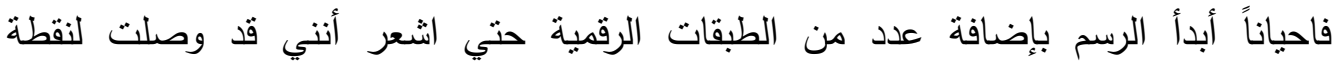


الانتهاء)(4). كما في لوحة رقم ( r) ، وفي سؤال “ لجورجي فاسيلي " موسس هذا الاتجاه

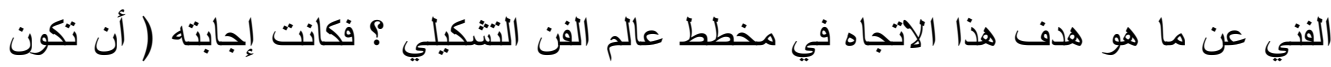
حركة ثورية ولكن غير جذرية ، مسموعة ولاكن ليس ذات صوت مزعج ، مؤثزة ولكن ليست

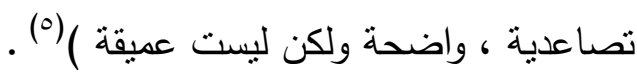
فالعمل الفني بالنسبة لفناني الطاقة هو كلام من القلب ، والبه ، وجسر من الالوان والطاقة يعبر

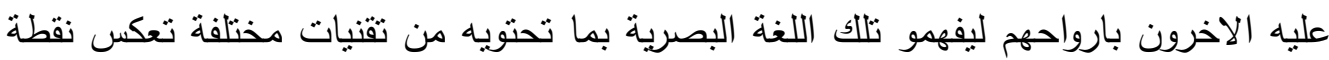

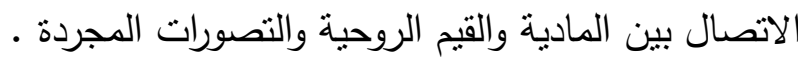
وفن الطاقة لا يقتصر علي اسلوب محدد ولكنه يشتمل علي العديد من الحركات

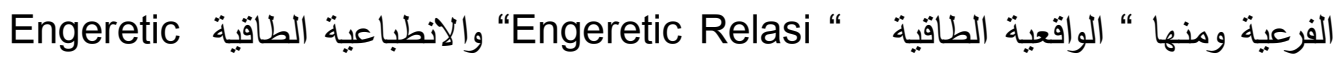
Impressionism " ، "والسريالية الطاقية Engeretic Ssrrealism"، “والتعبيرية

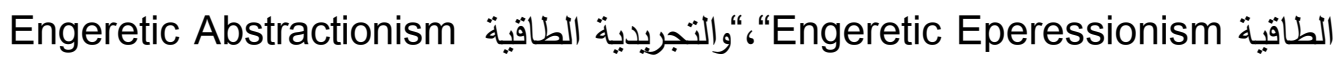

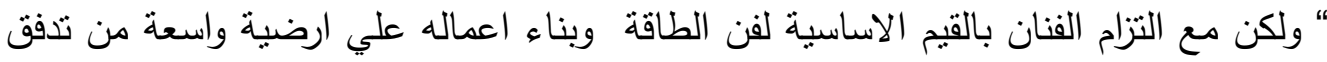

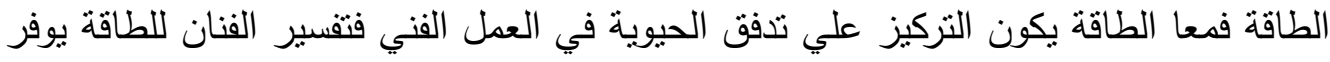

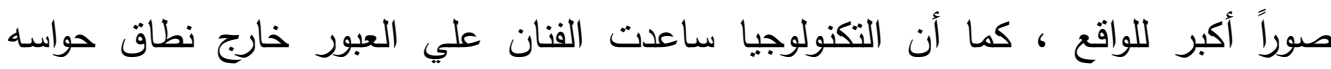
والثعور بالدجال المغناطيسي ، والكهرومغناطيسية ، والجاذبية .

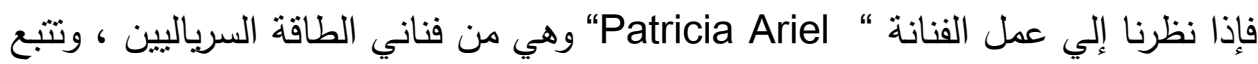

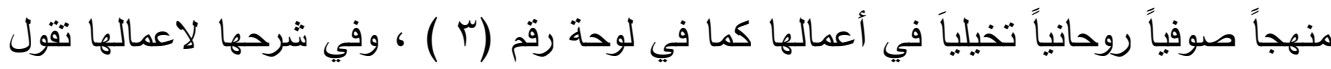

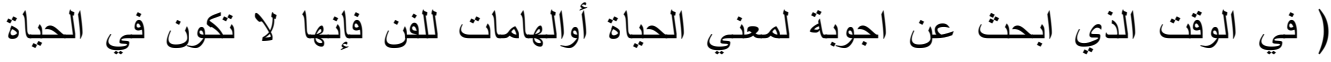
الانيوية العادية ولكني ابحث عنها في العالم الا محدود ، حيث نري في هذا العاء العالم الاماكن

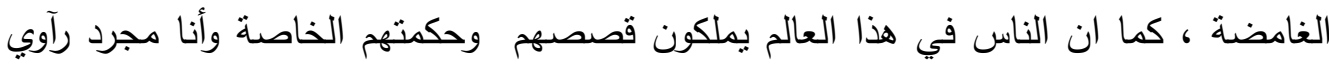
لهذه القصص ، فانا اعتقد أن أعمالي هي حديث القلب ، وجسر من الألوان والطاقة ، ومرآة

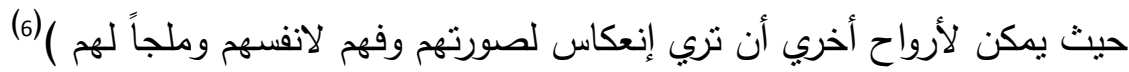

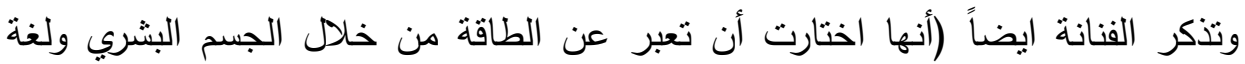

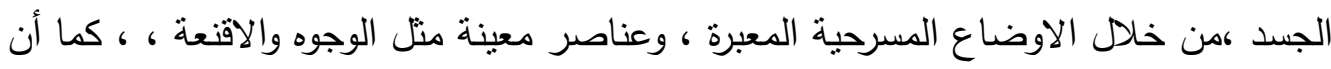

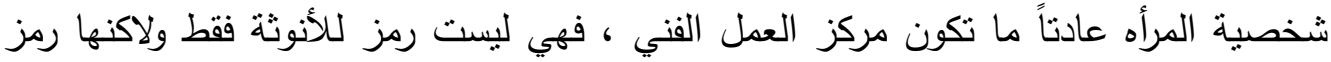

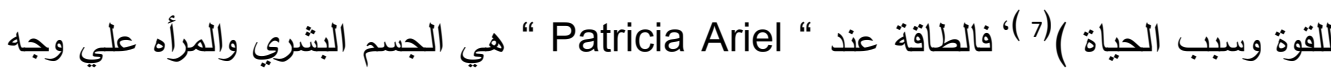

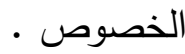
بينما يري الفنان “ Dan Bunea” الطاقة من خلال الحياة والتعبير عنها بالألوان المهنته والحركة التي لها تأثثر علي المشاهد ، فهي تستفزه وتملئه بالطاقة صورة رقم (ع ) ويعلق الفنان 
علي اعماله ( أريد للمشاهد أن يستمتع وأن برتبط عاطفياً مع اللوحة ويقتحم عالمها الملون ، الإنه

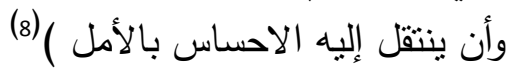

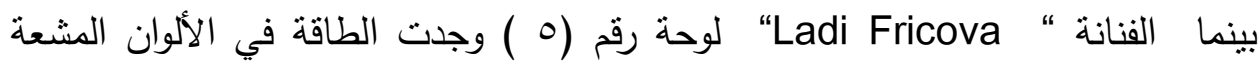

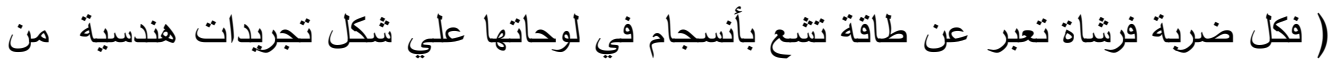

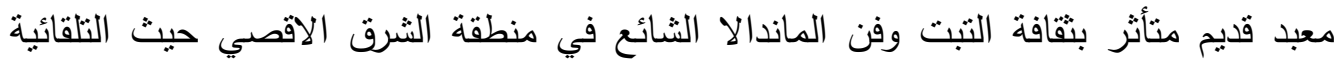

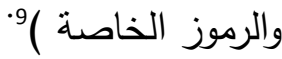

فالباحث من خلال هذا الاتجاه الفني المعاصر قد بعطي الفرصة للطيلاقة والمرونة

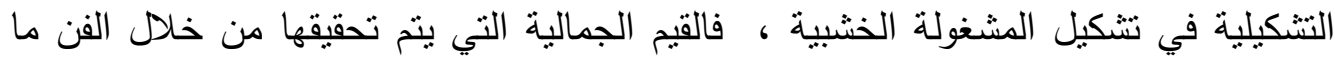

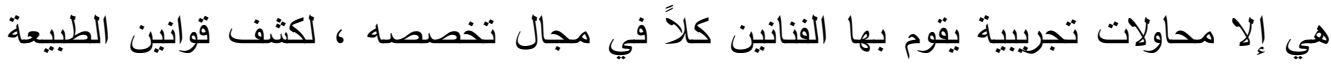

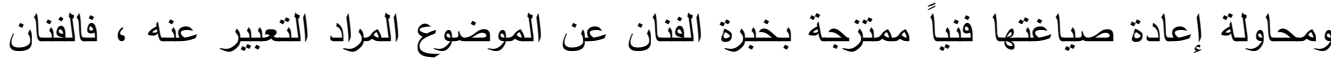

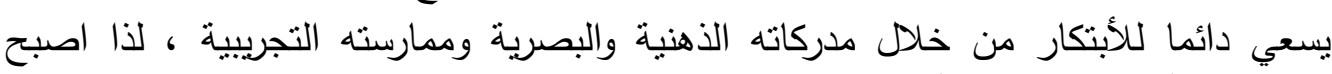

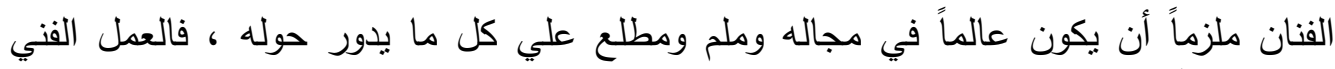

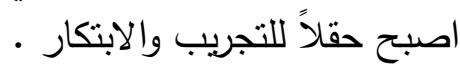
فالفنان المعاصر يبحث عما يوجد خلف المظهر الخارجي للاشياء لكى يقوم ببناء وتكوين الاشكال والتركيبات الفنية الجديدة على اساس خبراته الذاتية ورؤيته الموضوعية

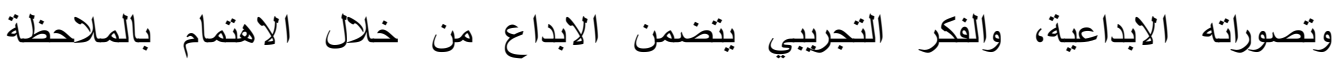

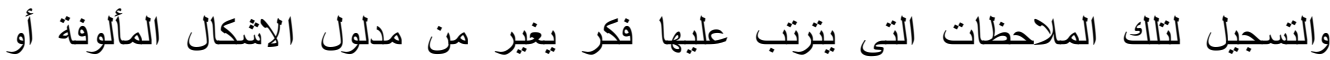
المعانى التقليدية . وخامة الخشب من الخامات التي تتميز بسهولة التشكيل وتقبلها لإجراء العمليات التقنية المختلفة ، والتي يمكن أن تساهم في تحقيق القيم الجمالية للمشغولة الخثبية ، مما يتيح للفنان

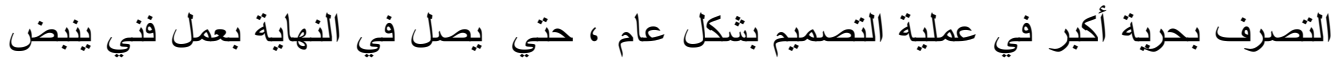

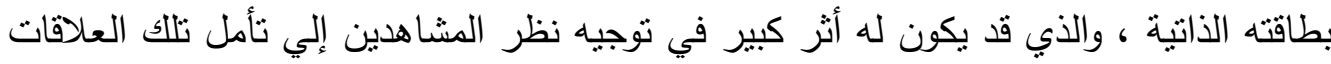

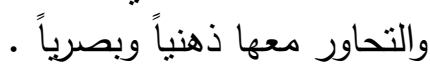

فمن خلال دراسة بعض الاعمال لفناني الطاقة، يمكن الكثف عن القيم الجمالية للمشغولة

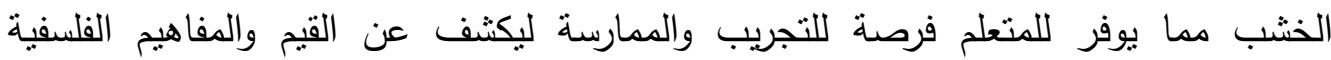

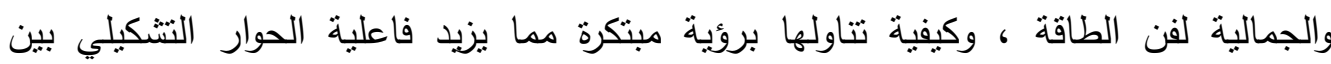

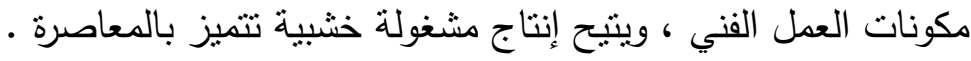

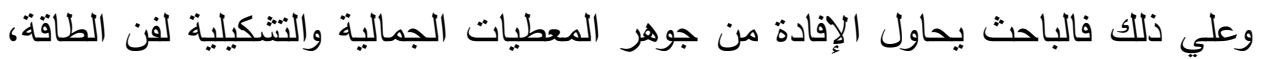

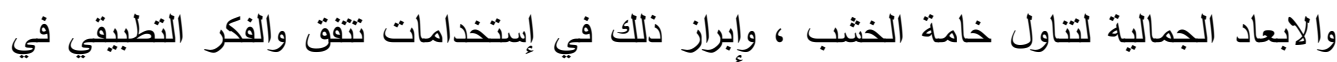

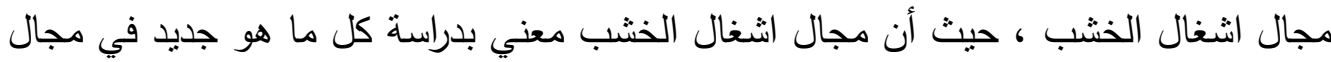

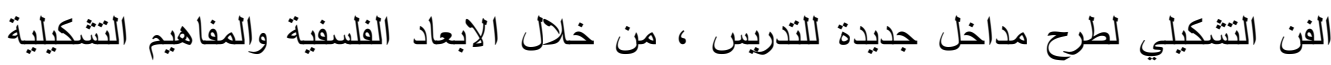

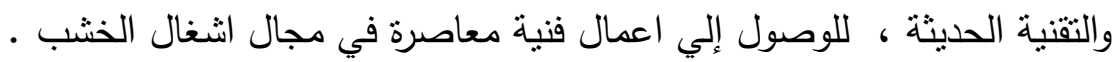


ومن هنا فالباحث يحاول تقديم دراسة تهتم بعرض الحلول الفنية للصياغات التشكيلية

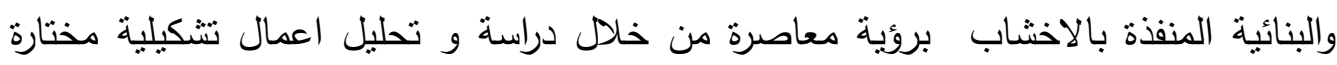

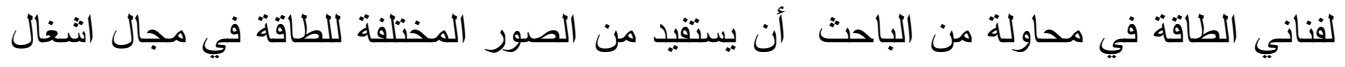

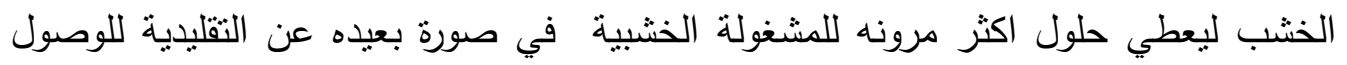

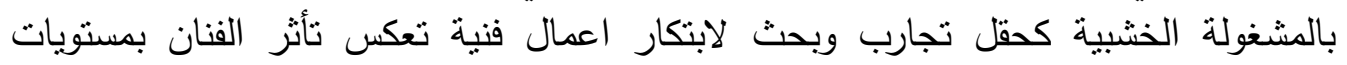
مختلفة من الطاقة .

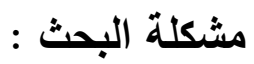

تتأثز العمليات الإبتكارية أثناء أنتاج الششغولة الخشبية بعوامل عديدة ، كالعوامل التقنية

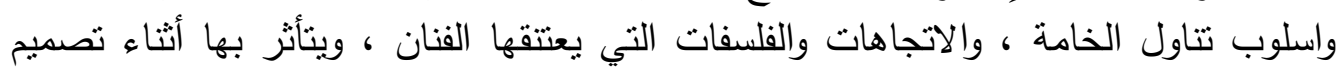

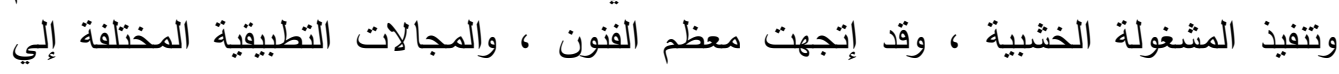

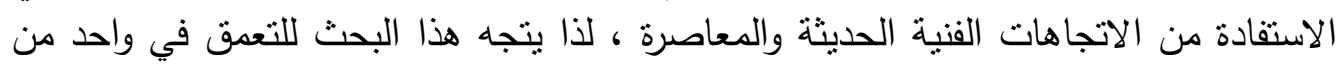

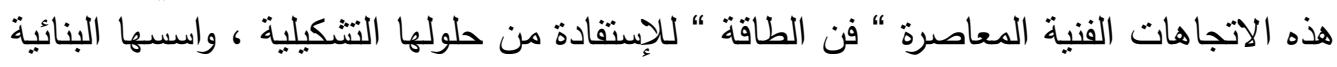

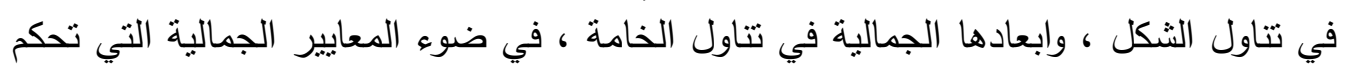

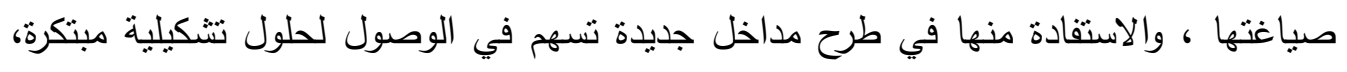

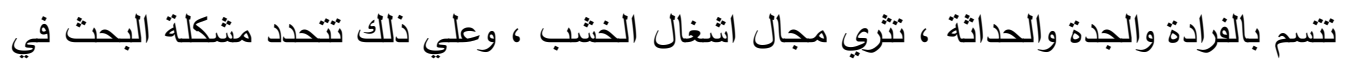
التساؤل التالي : بالني والجي

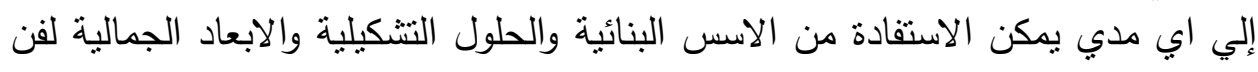

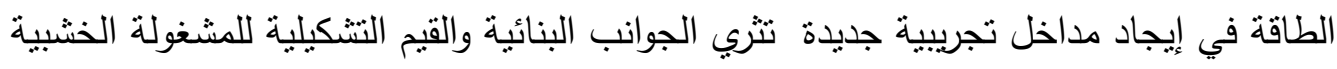

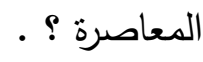

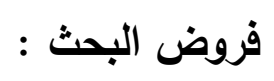

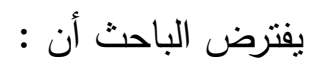

- يمكن طرح مداخل تجريبية لإثراء مجال اثغال الخشب بالإستفادة من الحلول التشكيلية

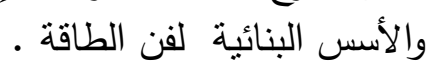
- يمكن إضافة مداخل تجريبية جديدة لمجال الشغال الخشب لطبالية بالإفادة من المفاهيم الجمالية والفلسفية لفن الطاقة . - يمكن الاستفادة من مفاهيم وقوانين فن الطاقة في إيجاد صياغات فنية وتشكيلية مبتكرة تثرى مجال اشغال الخشب .

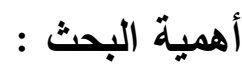

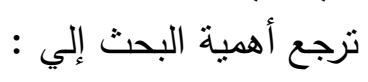
- محاولة اكتشاف مداخل تجريبية جديدة لتطوير العملية التدريسية بمجال أثغال الخشب بما

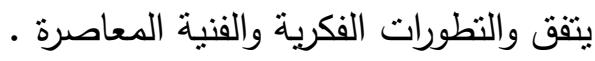
- - إضافة اتجاه فني جديد إلي مجال اشغال الخشب كمدخل لإثراء الجانب الإبداعي . 


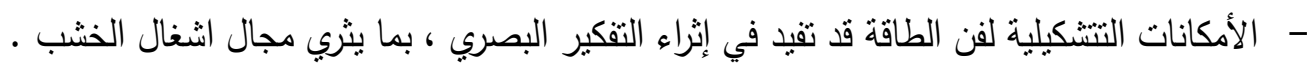

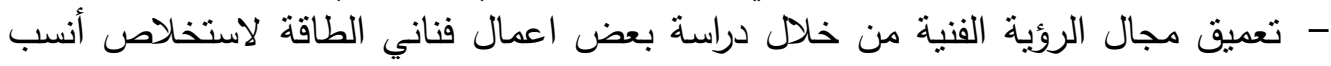
الحلول التشكيلية لإثراء مجال اشغال الخشب بن برؤية معاصرة ـ

$$
\text { أهداف البحث : يهدف البحث إلي : }
$$

- إثراء مجال اشغال الخشب جمالياً من خلال دراسة المفاهيم الفلسفية والجمالية لفن الطاقة .

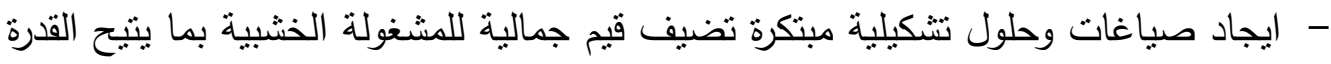

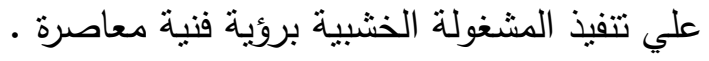
-

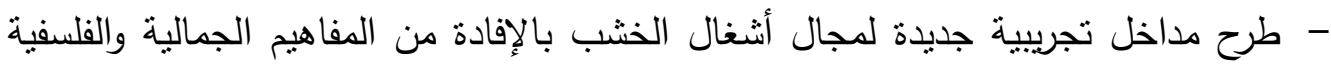

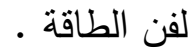
حدود البحث :

$$
\text { يقتصر البحث علي : دالبه : }
$$

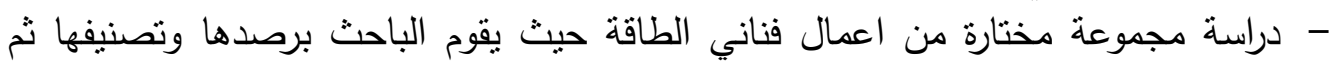

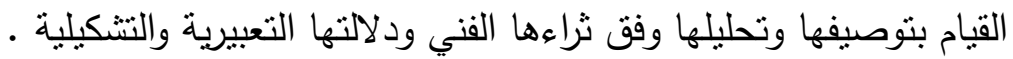
- استخدام تقنيات وأساليب التشكيل المختلفة في اشغال الخشب وكذاء ودلك الاخشاب بأنواعها وأنشكالها المختلفة

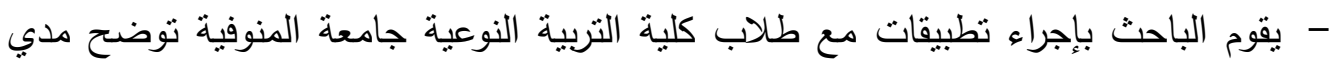
الإستفادة من هذه الدراسة في إنتاج مشغولات خشبية معاص كاصنة كرة كمدخل جديد في مجال

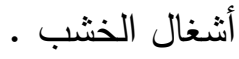

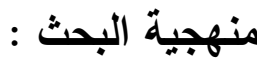
يتبع البحث في هذه الدراسة المنهج الوصفي التحليلي والمنهج التجربي من خلال.

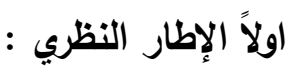
- دراسة تحليلية لمختارات من أعمال فناني الطاقة وذلك لإستخلاص المفاهيم الفلسفية

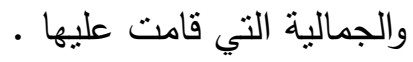
- بيان مدي الاستفادة من المعالجات والحلول التثكيلية والاثر التجريبي لفناني الطاقة

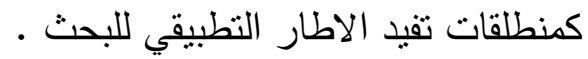

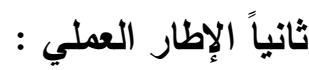

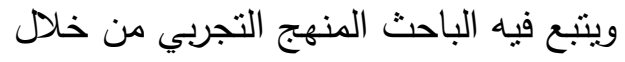
- تتفيذ تجارب استكثافية لتحليل الاساليب الادائية والحلول التقنية المناسبة لتنفيذ التصميمات

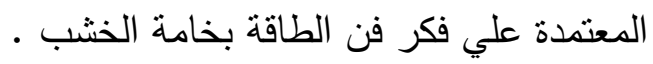


- يقوم الباحث بإجراء تجربة مع طلاب الفرقة الرابعة بكلية التربية النوعية جامعة المنوفية

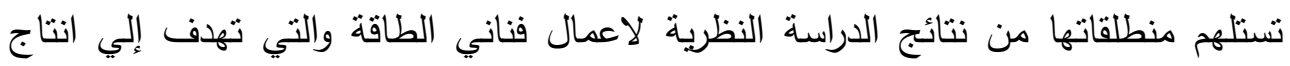

مشغولات خشبية بروئ معاصرة .

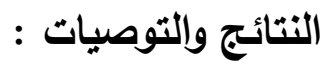

أولاً النتائج :

- دراسة الدفاهيم الجمالية والفلسفية لفن الطاقة بالتحليل والتجربب كان له أثر واضح في إثراء المشغولة الخشبية المعاصرة .

- أمكن إضافة مداخل تجريبية جديدة لمجال اشغال الخشب بالإفادة من المفاهيم الجمالية والفلسفية لفن الطاقة

- تقيد دراسة فن الطاقة إلي اتاحة المجال للخروج بالمشغولة الخشبية من نطاق التصميمات

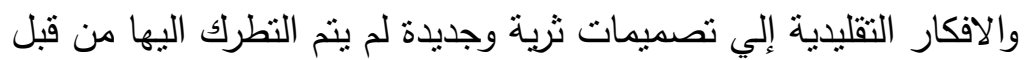

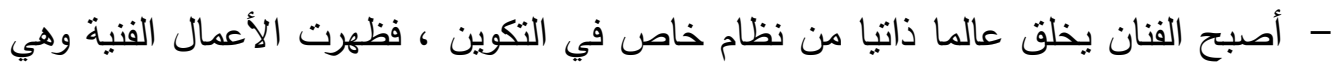

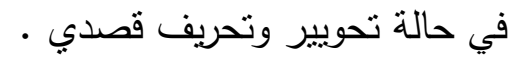

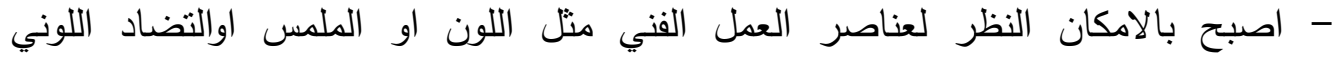

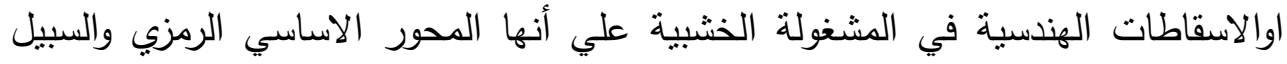

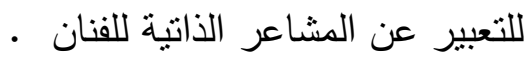
ثانياً التوصيات : لثنير :

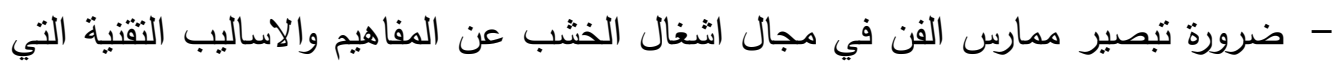

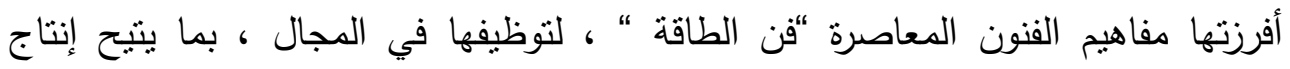

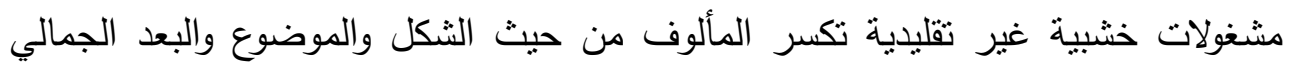
والتعبيري. - ضرورة تضمن المناهج الدراسية أساليب النتكيل التي تجمع بين المجالات المختلفة في

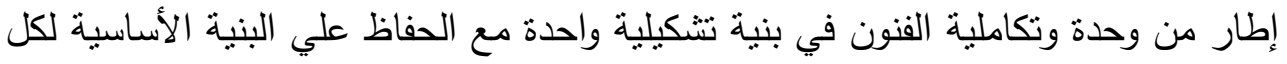

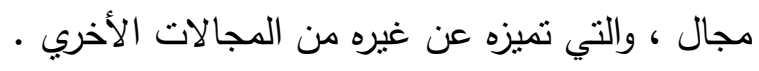

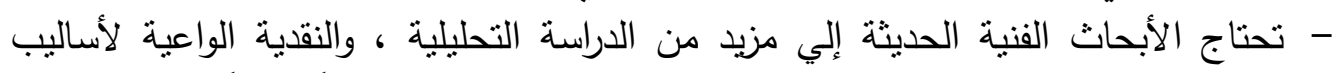

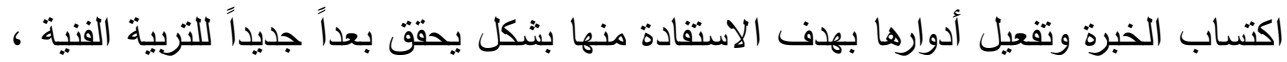

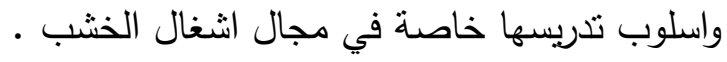




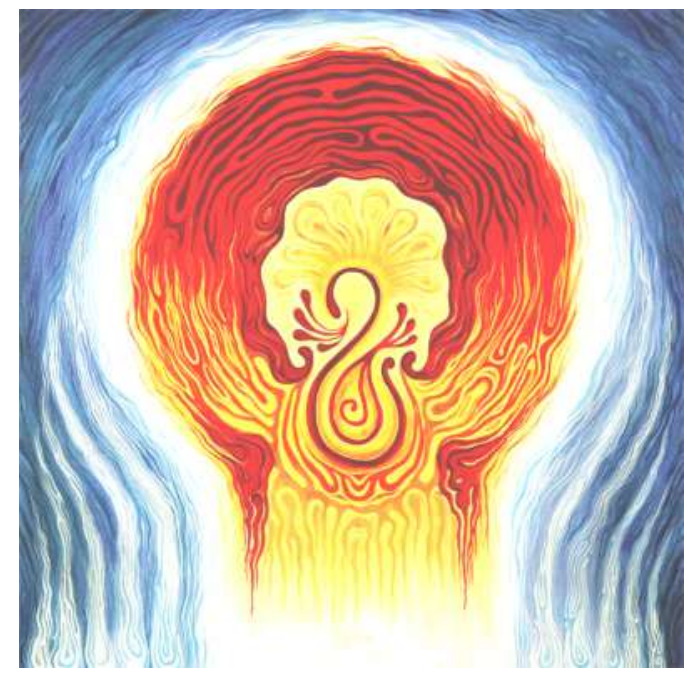

لوحة رقم (1) Ladi Fricova

www. Energyartmovement.org

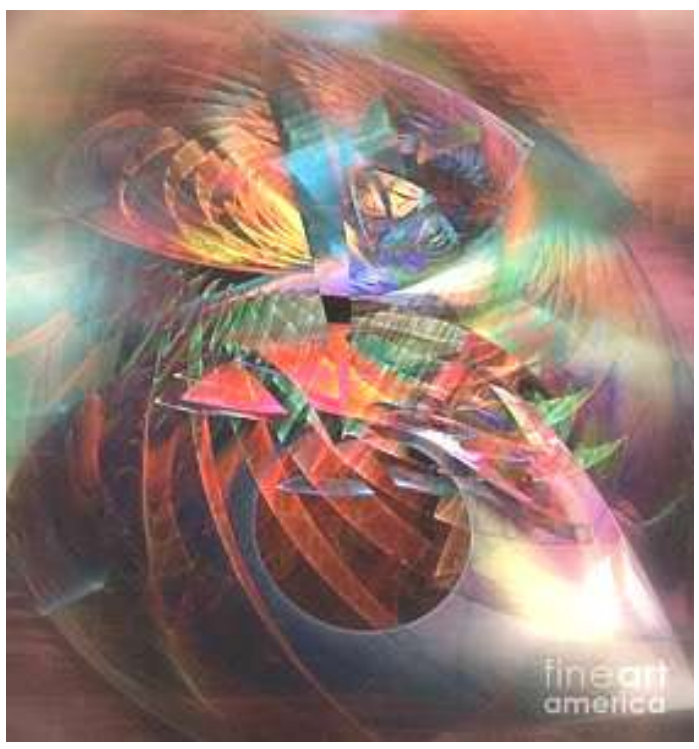

Helene Kippert (r) لوحة رقم

fineartamerica.com 


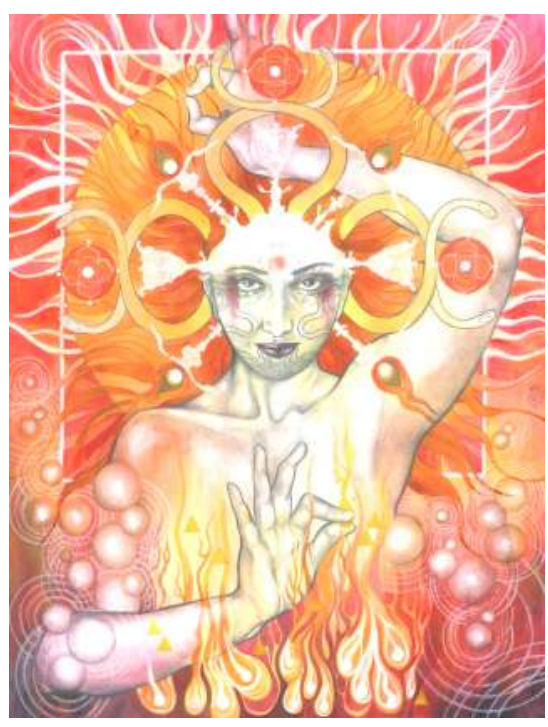

" Patricia Ariel " لوحة رقم (r)

http://art.patricia-ariel.com

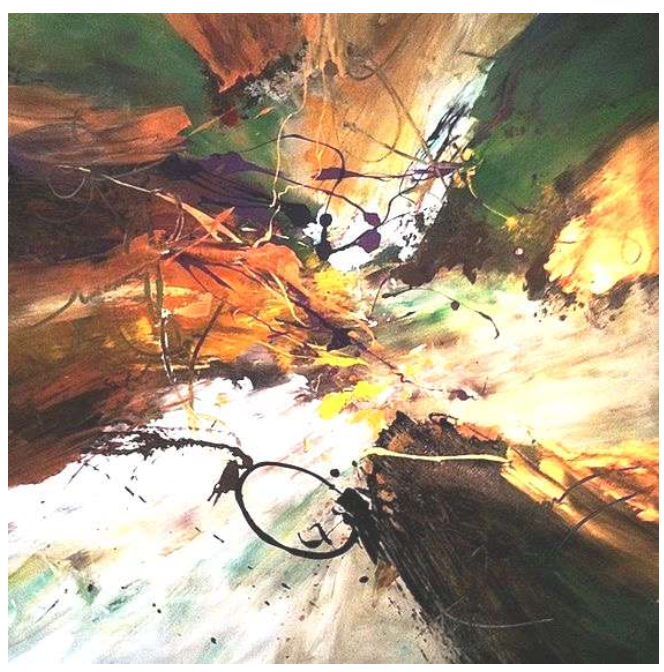

"Dan Bunea ") لوحة رقم (£)

https://www.pinterest.com 


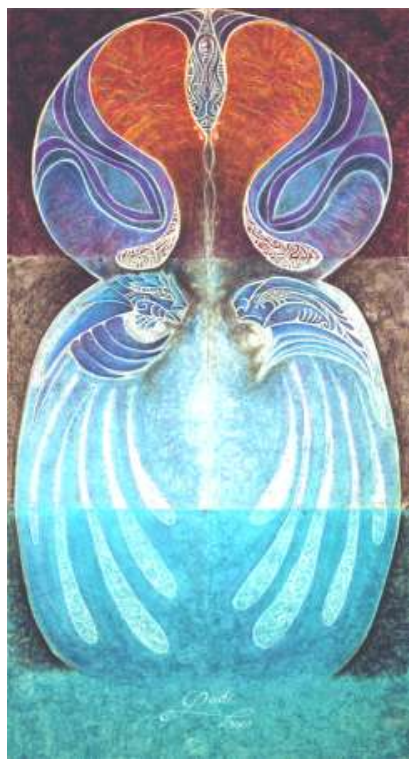

"Ladi Fricova " لوحة رقم(0)

Energy Art Movement's most recent Flickr photos

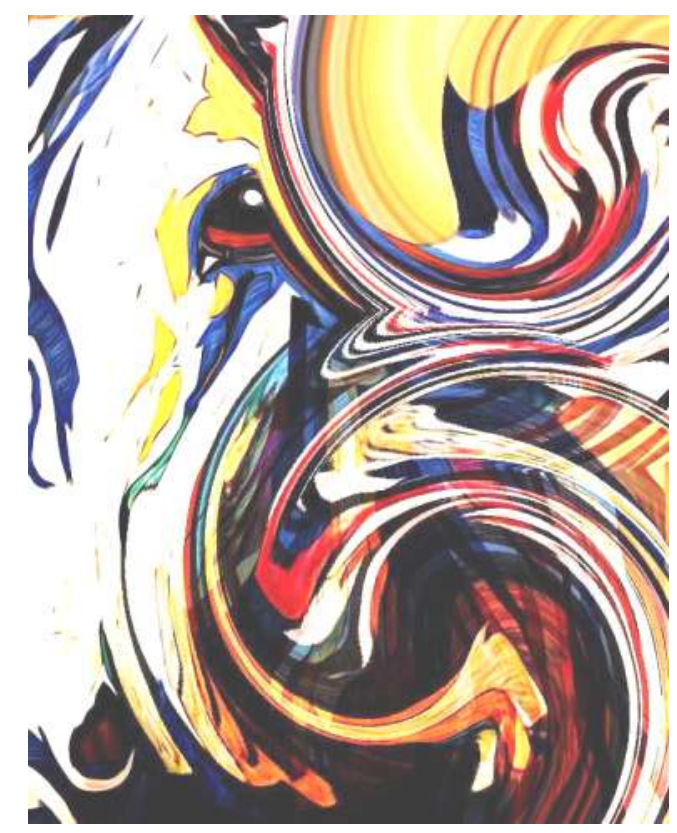

عمل الطالب محمد سمير - الفرقة الرابعة 


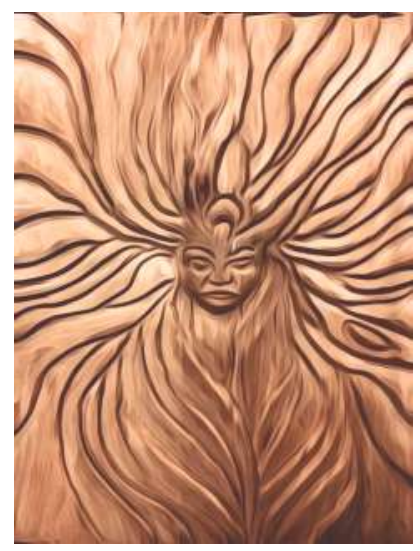

عمل الطالبة رانيا عبد العزيز الفرقة الرابعة

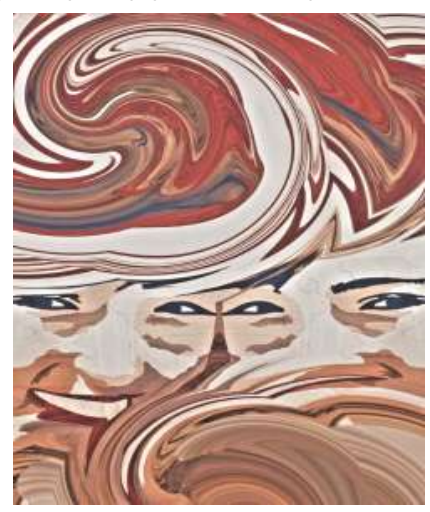

عمل الطالب محمد عبد السيد عبد الوارث- الفرقة الرابعة

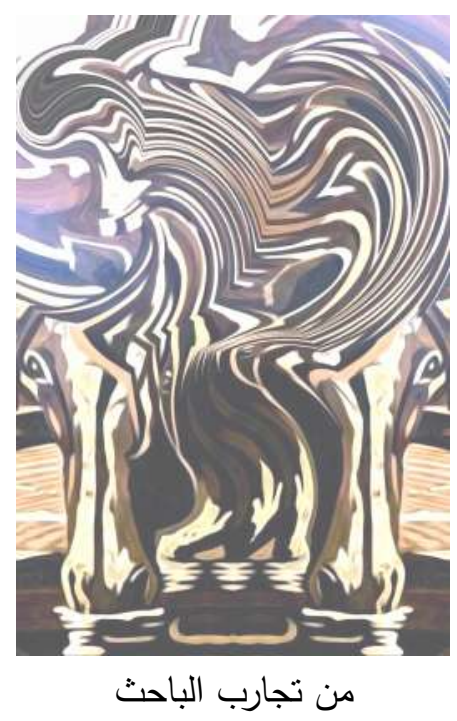




$$
\text { مصادر البحث: أولا : الكتب والمراجع العربية: }
$$

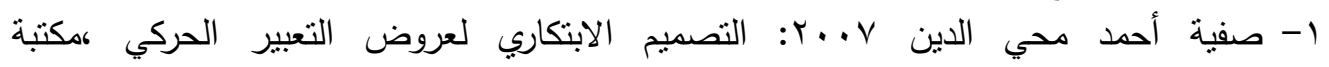
الانجلوالمصري، القاهرة

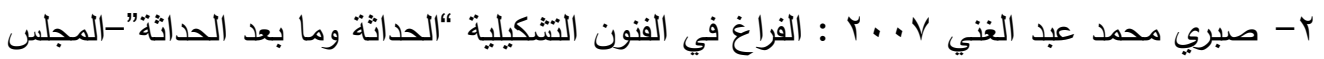
الاعلي للتقافة ، القاهرة

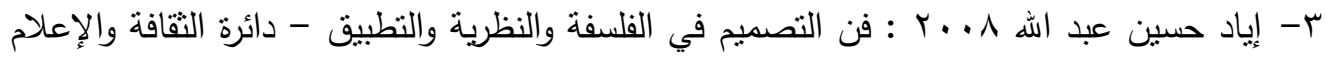

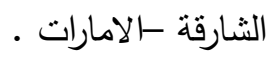

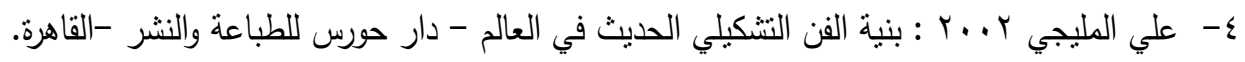

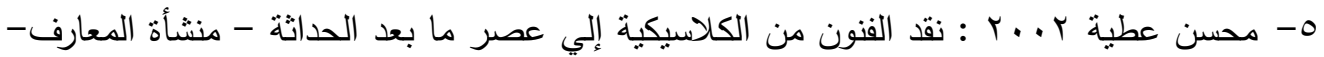

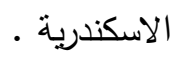

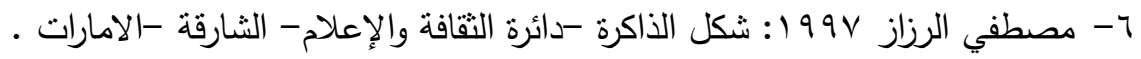

ثانياً : البحوث والرسائل العلمية

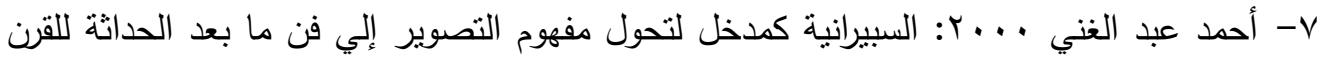

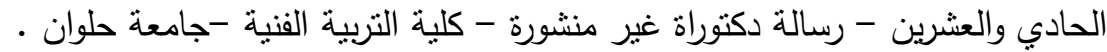

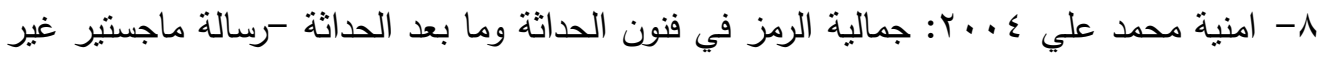

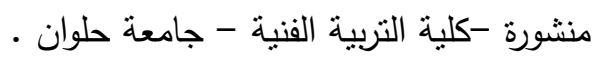

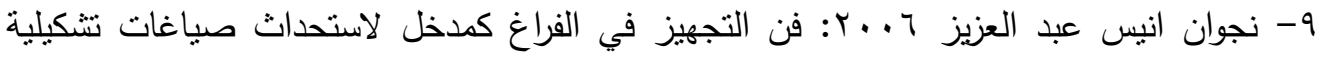

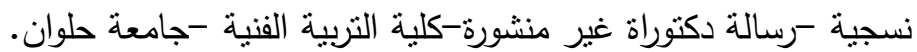

$$
\text { ثالثاً:المراجع الاجنبية والنترنت : }
$$

10-William Blake 2011 : "Energy is eternal delight." Essence of Balance Berkeley,unv.of California press,p19

11-Giorgio Vaselli (EAM Organizer) 2011 - The Energy Spectrum 2011 by the Energy Art Movement Curated - Kate Cherry (MMA Director), Joe MacGown (EAM Representative -

12- Helene Kippert - 2007 : Fractal worlds: Energy art for the New Earth -ltdWest Australian p36

13-patricia .ariel2007 :"Myth, Magic \& Lore -http://art.patricia-ariel.com

14- Patricia Ariel 2008:Myth, Magic and Lore" at Bash Contemporary- Mc Craw Hill,new york-p9

15- Dan Bunea - 2008 :Energy art for the New Earth - www.danbunea.com dan.bunea@gmail.com

16- Ladi Fricova 2009 :Energy Art Movement -higher education new york ,p 48

17- Mary .Stewart 2012 :Launching the Imagination, higter education, New york ،

2 -Giorgio Vaselli (EAM Organizer) 2011 - The Energy Spectrum 2011 by the Energy Art Movement Curated - Kate Cherry (MMA Director), Joe MacGown (EAM Representative- 
meridianmuseum-mississippi )

3- William Blake 2011: : "Energy is eternal delight." Essence of Balance -

Berkeley,unv.of California press,p19

4- Helene Kippert - 2007 : Fractal worlds: Energy art for the New Earth -ltd- West

Australian p36.

5 - Interview in Sublime Magazine - July - September -2010 Issue

6- :"Myth, Magic \& Lore: /2007: $\quad$ ariel.-patricia - -http://art.patricia-ariel.com

7 - Patricia Ariel 2008 :Myth, Magic and Lore" at Bash Contemporary- Mc Craw

Hill,new york-p9

3 - www.danbunea.com h-Dan Bunea - 2008 :Energy art for the New Eart dan.bunea@gmail.com

4 - Ladi Fricova 2009 :Energy Art Movement -higher education new york ,p 48. ${ }^{9}$ 